\title{
The Effect of the Radial Pressure Gradient in Protoplanetary Disks on Planetesimal Formation
}

\author{
Xue-Ning Bai \& James M. Stone \\ Department of Astrophysical Sciences, Princeton University, Princeton, NJ, 08544 \\ xbai@astro.princeton.edu, jstone@astro.princeton.edu
}

\begin{abstract}
The streaming instability (SI) provides a promising mechanism for planetesimal formation because of its ability to concentrate solids into dense clumps. The degree of clumping strongly depends on the height-integrated solid to gas mass ratio $Z$ in protoplanetary disks (PPDs). In this letter, we show that the magnitude of the radial pressure gradient (RPG) which drives the SI (characterized by $q \equiv \eta v_{K} / c_{s}$, where $\eta v_{K}$ is the reduction of Keplerian velocity due to the RPG and $c_{s}$ is the sound speed) also strongly affects clumping. We present local twodimensional hybrid numerical simulations of aerodynamically coupled particles and gas in the midplane of PPDs. Magnetic fields and particle self-gravity are ignored. We explore three different RPG values appropriate for typical PPDs: $q=0.025,0.05$ and 0.1 . For each $q$ value, we consider four different particle size distributions ranging from sub-millimeter to meter sizes and run simulations with solid abundance from $Z=0.01$ up to $Z=0.07$. We find that a small RPG strongly promotes particle clumping in that: 1) At fixed particle size distribution, the critical solid abundance $Z_{\text {crit }}$ above which particle clumping occurs monotonically increases with $q$; 2) At fixed $Z$, strong clumping can occur for smaller particles when $q$ is smaller. Therefore, we expect planetesimals to form preferentially in regions of PPDs with a small RPG.
\end{abstract}

Subject headings: diffusion — hydrodynamics — instabilities — planetary systems: protoplanetary disks — planets and satellites: formation — turbulence

\section{Introduction}

Planetesimals are super-kilometer sized bodies that are the building blocks of planets (Safronov 1969; Chiang \& Youdin 2009), yet their formation has long been a mystery. Millimeter and centimeter sized particles are routinely observed in protoplanetary disks (PPDs) (Wilner et al. 2005; Rodmann et al. 2006; Natta et al. 2007; Lommen et al. 2010), but parti-

cles with larger size seem difficult to form by coagulation (Blum \& Wurm 2008; Güttler et al. 2009). Solids close to meter size further suffer from rapid radial drift due to the negative radial pressure gradient (RPG) in PPDs (Weidenschilling 1977). The gravitational instability 
(GI) scenario of planetesimal formation (Goldreich \& Ward 1973) also has difficulties, because even without an external source of turbulence, the Kelvin-Helmholtz instability (KHI) generated from the dusty midplane layer prevents the onset of GI unless the local heightintegrated solid to gas mass ratio ( $Z$, hereafter referred to as solid abundance) is about an order of magnitude above solar metallicity (Weidenschilling 1980; Sekiva 1998; Youdin \& Shu 2002).

It was found recently that the drag interaction between solids and gas leads to a powerful instability (Goodman \& Pindor 2000). This "streaming instability" (SI, Youdin \& Goodman 2005) results in spontaneous particle clumping from the equilibrium state between solids and gas (Nakagawa et al. 1986). Numerical simulations demonstrate that the non-linear saturation of the SI concentrates particles into dense clumps (Johansen \& Youdin 2007), promoting planetesimal formation by collective particle self-gravity, bypassing the metersize barrier. Indeed, Johansen et al. (2007, 2009) showed that planetesimals with sizes of a few hundred kilometers form rapidly by the SI from centi-meter to decimeter sized pebbles and rocks, consistent with constraints from the asteroid belt (Morbidelli et al. 2009).

This letter complements our previous work on the dynamics of particle and gas in the midplane of PPDs (Bai \& Stone 2010b, hereafter BS10). We consider a wide size distribution of particles ranging from sub-millimeter up to meter sizes as an approximation for the outcome of dust coagulation in PPDs (Birnstiel et al. 2010; Zsom et al. 2010). External sources of turbulence such as magnetorotational instability are ignored, as appropriate for the dead zones in PPDs (Gammie 1996; Stone et al. 2000; Bai \& Goodman 2009), while SI is the main source of turbulence due to particle settling. Particle self-gravity is ignored in our simulations, as we focus on the precursor of planetesimal formation: particle clumping.

The SI is powered by the RPG in the gaseous disk. The RPG reduces the gas orbital velocity (in the absence of solids) by a fraction $\eta$ of the Keplerian velocity $v_{K}=\Omega r$, and is characterized by

$$
q \equiv \eta v_{K} / c_{s}=\eta r / H_{g}
$$

where $c_{s}$ is the isothermal sound speed, and $H_{g}=c_{s} / \Omega$ is the scale height of the gaseous disk. In BS10, we assumed $q=0.05$ throughout, while in reality $q$ depends on the parameters of, and location in, the disk. The strength of the SI turbulence scales with the RPG, which in turn affects the particle-gas dynamics in the disk midplane. In particular, we show in this letter that particle clumping strongly depends on the RPG, which has important implications for planetesimal formation.

\section{Simulations}

We perform two-dimensional (2D) hybrid simulations of gas and solids using the Athena code (Stone et al. 2008; Bai \& Stone 2010a), where gas is treated as a hydrodynamical fluid (without magnetic field) on an Eulerian grid, and the solids are treated as superparticles, 
each representing a swarm of real particles. We model a local patch of the PPDs using the shearing sheet approximation. The dynamical equations are written in a reference frame corotating at the Keplerian frequency $\Omega$ at fiducial radius $r$. We assume axisymmetry, and the simulations are performed in the radial-vertical $(x-z)$ plane, where $\Omega$ is along the $z$ direction and $x$ points radially outward. The particles are coupled to the gas via aerodynamic drag, characterized by the stopping time $t_{\text {stop }}$, with momentum feedback included. The gas is assumed to be isothermal, with midplane density $\rho_{g}$. Vertical gravity $g_{z}=-\Omega^{2} z$ is included for both particles and the gas.

The dynamical equations and simulation setup are identical to those in BS10. Specifically, we consider a particle size distribution that is discretized into a number of particle size bins, with each bin covering half a dex in $\tau_{s} \equiv \Omega t_{\text {stop }}$, bounded by the minimum and maximum stopping time $\tau_{\min }$ and $\tau_{\max }$. In what follows, we label our simulations with names of the form Rmn, where $m, n$ are integers obtained by $\tau_{\min }=10^{-m}$ and $\tau_{\max }=10^{-n}$, thus run Rmn uses $2(m-n)+1$ particle size bins (or particle species). We use a variety of grid resolution and box sizes to capture the fastest growing modes of the SI, and we use $10^{5}$ particles per species in all our simulations. We assume uniform mass distribution across all particle size bins, with total solid abundance $Z$. As in BS10, we consider four groups of runs, R41, R21, R30 and R10.

For each particle size distribution, we perform simulations with three different values of the RPG parameter: $q=0.025,0.05$ and 0.10. According to equations (4) and (7) in BS10, the dependence of $q$ on disk parameters such as temperature and mass is relatively weak, thus our range of $q$ covers a large parameter space of disk models. For each set of runs, we perform a series of simulations with different $Z$ values, starting at $Z=0.01$ and increasing the value by 0.01 for each new run, until strong particle clumping occurs or $Z=0.07$. Our simulation run parameters are summarized in Table 1. They are identical to the 2D run parameters in BS10, but use different values for $q$ and a larger range in $Z$. Since the natural length scale of the SI is $\eta r$, we use smaller (bigger) simulation box sizes for smaller (larger) $q$ values so that $\eta r$ is resolved by an equal number of grid cells in each series of runs. All simulations are run for at least $900 \Omega^{-1}$.

Three-dimensional (3D) simulation with the inclusion of the azimuthal dimension is necessary to capture the KHI (Chiang 2008; Barranco 2009), which is mainly caused by the vertical shear in the gas azimuthal velocity. Our simulations are 2D rather than 3D for two reasons. First, for our adopted particle size distribution, the turbulence generated from the SI stops particles from settling before the onset KHI (BS10), at least for the $q=0.05$ case. Second, in order to properly resolve the SI, relatively high resolution is required (Bai \& Stone 2010a), and particle clumping does depend on resolution 1 . Simulations in 3D with such high resolution are too costly due to the small box size (thus time step), especially for $q=0.025$.

\footnotetext{
${ }^{1}$ For example, Run R30Z3-3D in BS10 has no particle clumping in our standard resolution, but shows clumping at lower resolution.
} 


\section{Particle Clumping}

The simulations saturate in about 50-100 orbits. Particle settling triggers the SI, and particles with different stopping times are maintained at different heights determined by the balance between settling and turbulent diffusion. Most interestingly, SI efficiently concentrate particles into dense clumps when the solid abundance is sufficiently high (Johansen et al. 2009, BS10). In Figure 1 we plot the evolution of maximum particle density $\rho_{p, \max }$ for the R30 runs with different values of $q$ and $Z$. There is a clear dichotomy in the evolution: either the maximum particle density stays at a relatively small value $\left(\rho_{\mathrm{p}, \max } \lesssim 50 \rho_{g}\right)$, or it reaches as high as $10^{3} \rho_{g}$, indicative of strong clumping. A particle clump becomes gravitationally bound when its density exceeds the Roche density (see equation (18) of BS10), which also is of the order $10^{3} \rho_{g}$ for typical PPDs. Therefore, particle clumping is a prelude to planetesimal formation via gravitational collapse. For each value of $q$, the transition from non-clumping to clumping is sharp as $Z$ gradually increases, and we can define a critical solid abunance $Z_{\text {crit }}$, where strong particle clumping occurs for $Z>Z_{\text {crit }}$. Comparing different panels indicates that $Z_{\text {crit }}$ monotonically increases with $q$.

To better demonstrate the effect of the disk RPG on planetesimal formation, we plot the maximum particle density as a function of solid abundance $Z$ for each group of simulations with all values of $q$ in Figure 2, The maximum density is taken from the largest value of $\rho_{p, \max }$ over the last 20 orbits of the simulations, when all the runs are fully saturated. We take $\rho_{p}=500 \rho_{g}$ (which is the same order as the Roche density) as a rough indicator of planetesimal formation, as shown by the dotted lines. For each value of $q$, one can determine $Z_{\text {crit }}$ at the intersection of the dashed and corresponding solid lines. We see that for all four groups of runs, $Z_{\text {crit }}$ monotonically increases with $q$. Moreover, $Z_{\text {crit }}$ depends on $q$ more sensitively when the particle size is on average smaller. When $q=0.025, Z_{\text {crit }}$ is about 0.015 for all our four groups of runs. For $q=0.1$, run R41 does not show particle clumping below $Z=0.07$, for runs $\mathrm{R} 21$ and $\mathrm{R} 30 Z_{\text {crit }}$ is about 0.06 , while for runs $\mathrm{R} 10 Z_{\text {crit }}$ drops to below 0.05 .

Particle clumping is a highly non-linear effect due to the SI, and is more likely to develop when the average disk midplane solid to gas mass ratio $\epsilon$ is large. If one assumes $D$ to be the midplane vertical diffusion coefficient of the SI turbulence, particles with stopping time $\tau_{s}$

would settle to a layer with thickness of the order $H_{p} \approx \sqrt{D / \Omega \tau_{s}}$. The diffusion coefficient $D$ depends on the particle size distribution, $\epsilon \approx Z H_{g} / H_{p}$ and $q$. Without vertical gravity, the only length scale in the problem is $\eta r=q H_{g}$, thus $D \propto q^{2}$. If one assumes $D \propto \epsilon^{\alpha}$, we obtain by generalizing the toy model in BS10 (see their equation (16) and (17))

$$
H_{p} \propto Z^{\alpha /(\alpha+2)} q^{2 /(\alpha+2)} .
$$

Since one expects $\alpha<0$ for relatively large $\epsilon \gtrsim 1$, we see that $H_{p}$ not only depends sensitively on $Z$, as shown in BS10, it depends even more sensitively on $q$. This critical dependence makes the average particle to gas mass ratio $\epsilon$ at midplane quickly increases as $q$ decreases, promoting strong particle clumping, which explains the trend in Figure 2. The more sensitive 
Table 1: Run parameters.

\begin{tabular}{ccccccc}
\hline \hline Run & $q$ & $100 Z$ & $\tau_{\min }$ & $\tau_{\max }$ & $L_{x} \times L_{z}{ }^{1}$ & $N_{x} \times N_{z}{ }^{2}$ \\
\hline & 0.025 & $1 . .3$ & & & $0.05 \times 0.15$ & $256 \times 768$ \\
R41 & 0.05 & $1 . .5$ & $10^{-4}$ & $10^{-1}$ & $0.1 \times 0.3$ & $256 \times 768$ \\
& 0.1 & $1 . .7$ & & & $0.2 \times 0.4$ & $256 \times 512$ \\
\hline \multirow{2}{*}{ R21 } & 0.025 & $1 . .3$ & & & $0.05 \times 0.15$ & $256 \times 768$ \\
& 0.05 & $1 . .4$ & $10^{-2}$ & $10^{-1}$ & $0.1 \times 0.3$ & $256 \times 768$ \\
& 0.1 & $1 . .7$ & & & $0.2 \times 0.4$ & $256 \times 512$ \\
\hline \multirow{2}{*}{ R30 } & 0.025 & $1 . .3$ & & & $0.2 \times 0.3$ & $256 \times 512$ \\
& 0.05 & $1 . .3$ & $10^{-3}$ & 1 & $0.2 \times 0.3$ & $256 \times 384$ \\
& 0.1 & $1 . .7$ & & & $0.2 \times 0.3$ & $256 \times 384$ \\
\hline \multirow{2}{*}{ R10 } & 0.025 & $1 . .3$ & & & $0.2 \times 0.3$ & $256 \times 512$ \\
& 0.05 & $1 . .3$ & 10.1 & 1 & $0.2 \times 0.3$ & $256 \times 384$ \\
& 0.1 & $1 . .6$ & & & $0.2 \times 0.3$ & $256 \times 384$ \\
\hline \hline
\end{tabular}

${ }^{1}$ Domain size, in unit of gas scale height $H_{g}=c_{s} / \Omega$.

${ }^{2}$ Grid resolution.

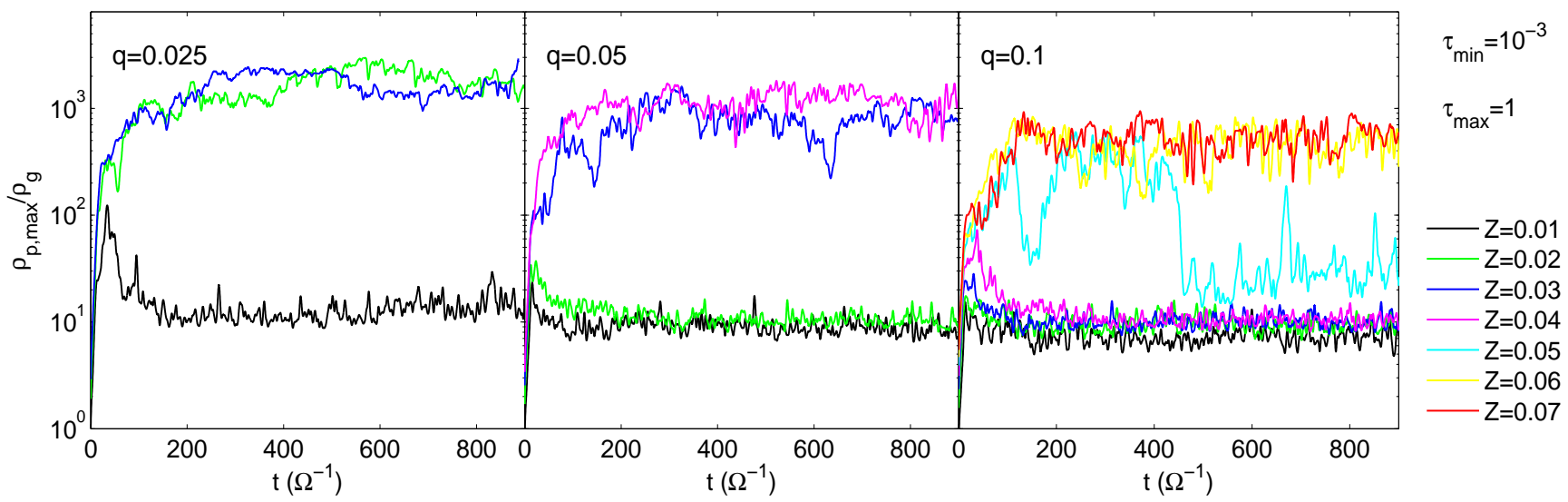

Fig. 1.- Time history of the maximum particle density in simulations with a range of particle sizes from $\tau_{s}=10^{-3}$ to one (runs R30). Left, middle and right panels show the results from $q=0.025,0.05$ and 0.1 respectively. Runs with different solid abundance $Z$ are labeled with different colors. 
dependence of $Z_{\text {crit }}$ on $q$ for smaller particles can be interpreted as a result of the power-law index $\alpha$ tending to be more negative for smaller $\tau_{s}$.

Our 2D simulations do not contain the azimuthal dimension, which suppresses KHI. Nevertheless, we can check whether KHI would occur before particles settle into a sufficiently thin layer to trigger strong SI by plotting the vertical profile of the Richardson number for simulations at critical solid abundance for all $q$ values, see Figure 3 . The Richardson number is calculated by the method described in $\S 3.1$ of BS10. Although the Richardson number alone does not determine the stability when Coriolis force (Gómez \& Ostriker 2005), and radial shear (Barranco 2009) are present, one may still take $R i_{\text {crit }}=0.1$ as an approximation for the critical value for instability (Chiang 2008; Lee et al. 2010). We see in Figure 3 that for most of these runs, the Richardson number across the disk is above the critical value, meaning that the system is $\mathrm{KH}$ stable at $Z_{\text {crit }}$. Moreover, as $q$ increases, the Richardson number at $Z_{\text {crit }}$ decreases. In particular, for R30 and R10 runs with $q=0.1$, the Richardson number at the disk midplane drops below 0.1, which may be subject to KHI. Therefore, the condition for strong clumping for these runs may be more stringent than shown in Figure 2 .

\section{Discussions and Conclusions}

In this letter, we have demonstrated that a small RPG strongly favors particle clumping and planetesimal formation via three effects. First, for a fixed particle size distribution, the critical solid abundance $Z_{\text {crit }}$ above which strong clumping occurs is reduced. Second, at fixed solid abundance, strong clumping occurs for smaller particles. Third, KHI is less likely to be triggered (and therefore will not suppress particle clumping) at $Z=Z_{\text {crit }}$. Moreover, a smaller RPG also implies smaller radial drift and collision velocities, promoting grain growth, strengthening our second point above. A small RPG also favors the GI scenario of planetesimal formation (relevant when all particles are strongly coupled to the gas) because there is less free energy available in the vertical shear 2 .

One caveat from our 2D simulations is that the condition for particle clumping is slightly more stringent in $3 \mathrm{D}$ than in $2 \mathrm{D}$ (BS10). Therefore, the values of $Z_{\text {crit }}$ obtained in this letter may be considered as a lower bound. Nevertheless, the conclusions in this letter are robust. In fact, Johansen et al. (2007, see their supplemental material) also found that planetesimal formation is eased with smaller pressure gradient using 3D simulations.

Combined with the results in BS10, we have shown that strong particle clumping is favored when there is: 1) a small RPG; 2) large solid abundance; 3) large solid size; and 4) a dead zone. These results indicate that planetesimals preferentially form in specific locations in PPDs. The global structure and evolution of PPDs is of crucial importance: it determines the RPG profile, the radial transport of particles which leads to enhancement of solid

\footnotetext{
${ }^{2}$ We expect the threshold abundance for GI to operate is larger than $Z_{\text {crit }}$.
} 


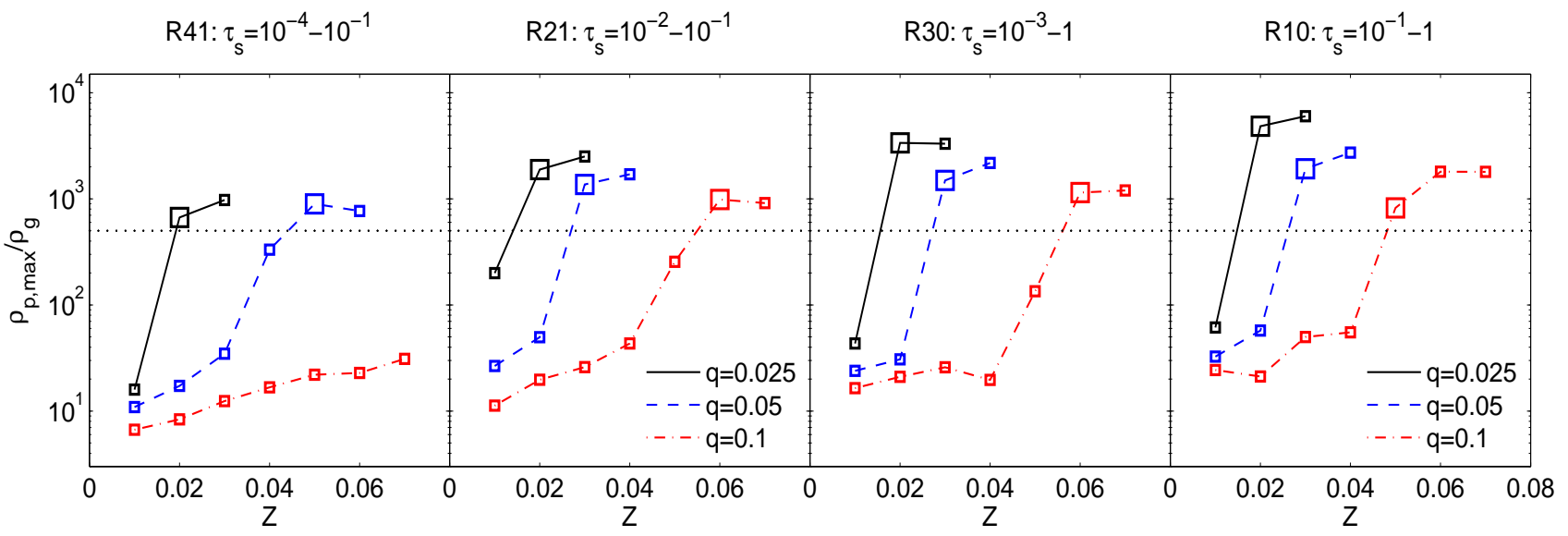

Fig. 2.- The maximum particle density that can be achieved by SI from all our simulation runs (for R41, R21, R30, R10 from left to right panels). In each panel, we plot the maximum particle density as a function of solid abundance $Z$ for each value of $q$ (black solid, blue dashed and red dash-dotted for $q=0.025,0.5$ and 0.1 respectively). The dotted line at $\rho_{p, \max }=500 \rho_{g}$ indicates the adopted threshold for strong particle clumping, and we use larger symbols to indicate the first runs that show strong clumping as $Z$ increases.

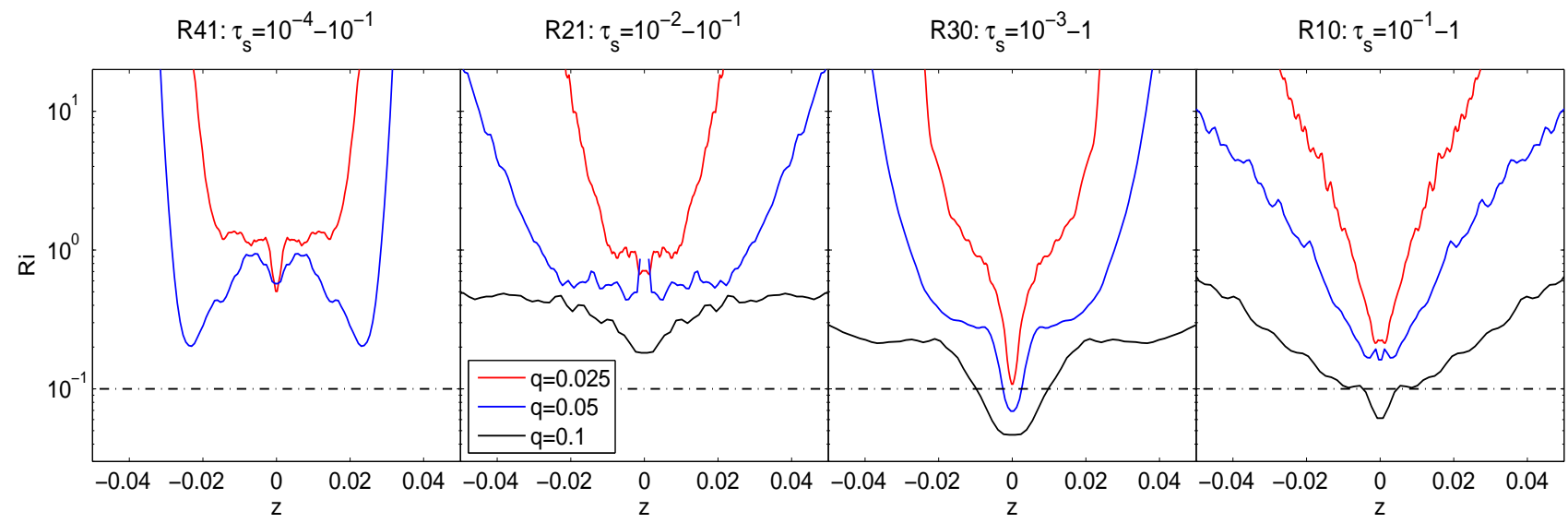

Fig. 3. - The Richardson number profile for all simulation runs (for R41, R21, R30, R10 from left to right panels) with solid abundance just above $Z_{\text {crit }}$ (the runs with enlarged symbols in Figure 21). Results from $q=0.025,0.05$ and 0.1 are shown in black solid, blue dashed and red dash-dotted curves respectively. The dotted line indicated an approximate estimate of the critical Richardson number $R i_{\text {crit }}=0.1$. 
abundance in the inner disk (Youdin \& Shu 2002), and grain coagulation that determines the particle size distribution. Recent models on the structure and evolution of PPDs (e.g., Jin \& Sui 2010; Zhu et al. 2010) generally show much more complicated structures than the simple MMSN or $\alpha$-disk models due to non-steady state accretion as well as the presence of dead zones. In addition, RPG may reach zero in local pressure bumps at the snow line (Kretke \& Lin 2007) or the inner edge of the dead zone (Dzyurkevich et al. 2010) which can also be the preferred site for planetesimal formation. These results all-together provide useful constraints on the initial conditions for the formation of planet embryos and planets (Kokubo \& Ida 2000, 2002).

We are grateful to D.N.C. Lin for helpful discussions. This work is supported by NSF grant AST-0908269. XNB acknowledges support from NASA Earth and Space Science Fellowship.

\section{REFERENCES}

Bai, X.-N. \& Goodman, J. 2009, ApJ, 701, 737

Bai, X.-N. \& Stone, J. 2010a, ApJ, submitted

—. ApJ, 2010b, submitted

Barranco, J. A. 2009, ApJ, 691, 907

Birnstiel, T., Dullemond, C. P., \& Brauer, F. 2010, ArXiv e-prints

Blum, J. \& Wurm, G. 2008, ARA\&A, 46, 21

Chiang, E. 2008, ApJ, 675, 1549

Chiang, E. \& Youdin, A. 2009, ArXiv e-prints

Dzyurkevich, N., Flock, M., Turner, N. J., Klahr, H., \& Henning, T. 2010, ArXiv e-prints

Gammie, C. F. 1996, ApJ, 457, 355

Goldreich, P. \& Ward, W. R. 1973, ApJ, 183, 1051

Gómez, G. C. \& Ostriker, E. C. 2005, ApJ, 630, 1093

Goodman, J. \& Pindor, B. 2000, Icarus, 148, 537

Güttler, C., Blum, J., Zsom, A., Ormel, C. W., \& Dullemond, C. P. 2009, ArXiv e-prints

Jin, L. \& Sui, N. 2010, ApJ, 710, 1179 
Johansen, A., Oishi, J. S., Low, M.-M. M., Klahr, H., Henning, T., \& Youdin, A. 2007, Nature, 448, 1022

Johansen, A. \& Youdin, A. 2007, ApJ, 662, 627

Johansen, A., Youdin, A., \& Mac Low, M. 2009, ApJ, 704, L75

Kokubo, E. \& Ida, S. 2000, Icarus, 143, 15

-. 2002, ApJ, 581, 666

Kretke, K. A. \& Lin, D. N. C. 2007, ApJ, 664, L55

Lee, A. T., Chiang, E., Asay-Davis, X., \& Barranco, J. A. 2010, ApJ, in preparation

Lommen, D. J. P., van Dishoeck, E. F., Wright, C. M., Maddison, S. T., Min, M., Wilner, D. J., Salter, D. M., van Langevelde, H. J., Bourke, T. L., van der Burg, R. F. J., \& Blake, G. A. 2010, ArXiv e-prints

Morbidelli, A., Bottke, W. F., Nesvorný, D., \& Levison, H. F. 2009, Icarus, 204, 558

Nakagawa, Y., Sekiya, M., \& Hayashi, C. 1986, Icarus, 67, 375

Natta, A., Testi, L., Calvet, N., Henning, T., Waters, R., \& Wilner, D. 2007, in Protostars and Planets V, ed. B. Reipurth, D. Jewitt, \& K. Keil, 767-781

Rodmann, J., Henning, T., Chandler, C. J., Mundy, L. G., \& Wilner, D. J. 2006, A\&A, 446, 211

Safronov, V. S. 1969, Evolution of the protoplanetary cloud and formation of the earth and the planets (Moscow: Nauka Press. Trans. NASA TTF 677, 1972)

Sekiya, M. 1998, Icarus, 133, 298

Stone, J. M., Gammie, C. F., Balbus, S. A., \& Hawley, J. F. 2000, in Protostars and Planets IV, eds. Mannings, V., Boss, A.P., Russell, S.S., (Tucson, U. Arizona Press), p589

Stone, J. M., Gardiner, T. A., Teuben, P., Hawley, J. F., \& Simon, J. B. 2008, ApJS, 178, 137

Weidenschilling, S. J. 1977, MNRAS, 180, 57

-. 1980, Icarus, 44, 172

Wilner, D. J., D’Alessio, P., Calvet, N., Claussen, M. J., \& Hartmann, L. 2005, ApJ, 626, L109

Youdin, A. N. \& Goodman, J. 2005, ApJ, 620, 459

Youdin, A. N. \& Shu, F. H. 2002, ApJ, 580, 494 
Zhu, Z., Hartmann, L., \& Gammie, C. 2010, ApJ, 713, 1143

Zsom, A., Ormel, C. W., Guettler, C., Blum, J., \& Dullemond, C. P. 2010, ArXiv e-prints 